\title{
Non-equilibrium statistics of a reduced model for energy transfer in waves
}

\author{
R. E. LEE DEVILLE \\ Courant Institute \\ PAUL A. MILEWSKI \\ University of Wisconsin, Madison \\ RICARDO J. PIGNOL \\ U. Nacional del Sur, Bahía Blanca, Argentina \\ ESTEBAN G. TABAK \\ Courant Institute \\ AND ERIC VANDEN-EIJNDEN \\ Courant Institute
}

\begin{abstract}
We study energy transfer in a "resonant duet" - a resonant quartet where symmetries support a reduced subsystem with only two degrees of freedom - where one mode is forced by white noise and the other is damped. We consider a physically motivated family of nonlinear damping forms, and investigate their effect on the dynamics of the system. A variety of statistical steady-states arise in different parameter regimes, including intermittent bursting phases, non-equilibrium states highly constrained by slaving among amplitudes and phases, and Gaussian and non-Gaussian quasi-equilibrium regimes. All of this can be understood analytically using asymptotic techniques for stochastic differential equations.
\end{abstract}

\section{Introduction}

Many wave systems in nature are best described in terms of Fourier modes, and the nonlinearities of the dynamics correspond to energy exchange amongst these modes. Often the dynamics is conservative in a large range of length scales (the inertial range), with forcing and dissipation acting only over a more restricted range. For example, ocean surface waves are thought to be initiated by the wind at small (capillary) scales, with the subsequent dynamics transferring the energy to longer scales. The main dissipation mechanism is wave breaking, which usually acts on much longer (gravity) waves that intermittently remove energy from the wave system.

For dispersive wave systems, such as surface ocean waves and internal waves in the atmosphere and ocean, the conservative energy transfer occurs mostly through resonant sets, typically triads or quartets. Weak

Communications on Pure and Applied Mathematics, Vol. 000, 0001-0027 (2003)

(C) 2003 John Wiley \& Sons, Inc.

CCC 0010-3640/98/000001-27 
turbulence (or wave turbulence) theory describes this weakly nonlinear conservative energy cascade through the resonant sets. The theory yields kinetic equations (where the energy exchange is through wave "collisions") for the evolution of the Fourier spectrum. Sometimes, special power law solutions of these equations with a prescribed flux of energy can be found $[6,2,1,7,11]$.

In idealized systems where the damping acts on infinitely small or large length-scales (the so-called inviscid limit), it is believed that the form of damping does not affect the spectrum. Away from this limit, however, the form of the forcing may affect the solution. For instance, developers of General Circulation Models for the Atmosphere and Ocean are well aware of the sensitivity of their models to the form of the parameterization of damping used. The purpose of the present work is to understand the effect of more realistic, nonlinear dissipation in the context of a simple model amenable to detailed analysis. To this end, we generalize the duet system introduced in [9]. The forms of dissipation that we consider include those consistent with wave breaking and therefore may prove useful in parameterizing wave breaking in more complex scenarios.

As in [9], the model emerges from isolating one resonant quartet in a general dispersive system, adding white noise forcing and dissipation, and then further reducing the system to just two complex degrees of freedom, by exploiting a symmetry. The reason to model the forcing through white noise is that this permits a complete control of the energy input, and hence also of the rate of energy transfer through the system, when it is in a statistically stationary state.

The resulting duet system has many analogies to more complex dispersive systems, but is of low enough dimension that it is quite amenable to numerical simulation. Furthermore, it is simple enough that it can be understood almost completely using theoretical tools. In spite of its simplicity, this model exhibits a rich variety of behaviors. In particular, it contains both Gaussian and non-Gaussian quasi-equilibrium states, in addition to states far from equilibrium, dominated by a maximally efficient energy transfer, with most of the dynamics slaved to the evolution of one single mode.

The paper is organized as follows.

In Section 2, we state the duet model we study in the paper, and show how it is related to a typical nonlinear PDE. In Section 3, we study some of the elementary statistical properties of the duet model. In Section 4 we describe a numerical study of the duet for various parameter values and 
show the existence of several parameter regimes. In Sections 5 and 6 we analyze the dynamical equations in two different asymptotic regimes and obtain the (sometimes approximate) distributions for the system. Finally, in Section 7 we summarize and suggest further work.

\section{The duet system}

We consider in this paper the resonant duet

$$
\left\{\begin{array}{l}
i \dot{a}_{1}=2 \gamma \bar{a}_{1} a_{2}^{2}+\sigma \dot{W}, \\
i \dot{a}_{2}=2 \gamma a_{1}^{2} \bar{a}_{2}-i \nu a_{2}\left|a_{2}\right|^{\beta},
\end{array}\right.
$$

where $a_{1}, a_{2}$ are two complex amplitudes, $\gamma>0, \sigma>0, \nu>0, \beta \geq 0$ are constants, and $W$ is a complex Weiner process. This is a prototypical model for energy transfer; we have two coupled modes, and we force one and damp the other with a view towards determining what proportion of the total energy remains in each mode. The parameter $\beta$ allows the damping to be nonlinear.

This is a generalization of the system studied in [9], which described the case $\beta=0$, corresponding, for instance, to a fluid's viscosity. The purpose of introducing a more general $\beta$ is to understand the role of nonlinearity in the dissipation mechanism, and as we show below this can lead to radically different qualitative behavior. There are several motivations to study this generalization. From a technical perspective, it is interesting to find physical systems that have equilibrium invariant measures which are non-Gaussian. From a physical perspective, dissipation in fluid problems is often brought about by nonlinear wave dynamics.

\subsection{Relation to the nonlinear Schrödinger equation}

As in [9], we start with a one-dimensional partial differential equation of the form

$$
i \frac{\partial \Psi}{\partial t}=L \Psi+\gamma|\Psi|^{2} \Psi+\text { forcing and damping, }
$$

where $L$ is a Hermitian linear operator with symbol $\hat{L}=\omega_{k}$. In the inertial range, this system behaves in a Hamiltonian manner as

$$
i \frac{\partial \Psi}{\partial t}=\frac{\partial H}{\partial \bar{\Psi}}, \quad H=\int d k \omega_{k}|\hat{\Psi}(k)|^{2}+\frac{\gamma}{2} \int d x|\Psi(x)|^{4} .
$$


Our goal here is to find a simple subsystem of (2.2) in which we can understand the mechanisms of energy transfer. First, we consider a single resonant quartet, i.e. a set of four wavenumbers $k_{j}$ such that the resonant conditions

$$
\begin{aligned}
k_{1}+k_{4} & =k_{2}+k_{3}, \\
\omega_{k_{1}}+\omega_{k_{4}} & =\omega_{k_{2}}+\omega_{k_{3}},
\end{aligned}
$$

are satisfied. If we then excite these four modes with $O(\epsilon)$ amplitudes, then the dynamics of $\hat{\Psi}_{k_{j}}$ can be approximated, up to leading order, by

$$
\hat{\Psi}_{k_{j}}(t)=\epsilon a_{j}(\tau) e^{-i\left(\omega_{k_{j}}-2 \epsilon \gamma m\right) t}
$$

with $\tau=\epsilon^{2} t, m=\sum_{k}\left|a_{k}\right|^{2}$ (the mass of the system), and the $a_{j}$ satisfy the resonant equations (see e.g. [5])

$$
\left\{\begin{array}{l}
i \dot{a}_{1}=2 \gamma \bar{a}_{4} a_{2} a_{3}-\gamma a_{1}\left|a_{1}\right|^{2}, \\
i \dot{a}_{2}=2 \gamma \bar{a}_{3} a_{1} a_{4}-\gamma a_{2}\left|a_{2}\right|^{2}, \\
i \dot{a}_{3}=2 \gamma \bar{a}_{2} a_{1} a_{4}-\gamma a_{3}\left|a_{3}\right|^{2}, \\
i \dot{a}_{4}=2 \gamma \bar{a}_{1} a_{2} a_{3}-\gamma a_{4}\left|a_{4}\right|^{2}
\end{array}\right.
$$

The last term in each equation comes from the "self-interaction" of each mode with itself (because of the trivial relation that $k_{j}+k_{j}=k_{j}+k_{j}$ ), and we will actually drop these terms in the sequel. This simplifies the calculations but does not change them qualitatively (cf. [9] where these terms are retained). We now drop the self-interaction terms in (2.3), noting that it retains its Hamiltonian structure, and obtain

$$
H=2 \gamma\left(\bar{a}_{1} a_{2} a_{3} \bar{a}_{4}+a_{1} \bar{a}_{3} \bar{a}_{3} a_{4}\right) .
$$

These equations satisfy the "Manley-Rowe" relations

$$
\frac{d\left|a_{1}\right|^{2}}{d t}=\frac{d\left|a_{4}\right|^{2}}{d t}=-\frac{d\left|a_{2}\right|^{2}}{d t}=-\frac{d\left|a_{3}\right|^{2}}{d t}
$$

from which follows the conservation of mass $m$, momentum $p$, and linear energy $e$, given as

$$
m=\sum_{j}\left|a_{j}\right|, \quad p=\sum_{j} k_{j}\left|a_{j}\right|^{2}, \quad e=\sum_{j} \omega_{k_{j}}\left|a_{j}\right|^{2} .
$$

With the fourth conserved quantity being the Hamiltonian itself, this makes this system integrable. This system can actually be solved analytically [5] but we do not need that here. 
Since we want to study energy transfer in (2.3), we consider the generalization

$$
\begin{aligned}
i \dot{a}_{1} & =2 \gamma \bar{a}_{4} a_{2} a_{3}+\sigma \dot{W}_{1}(t), \\
i \dot{a}_{2} & =2 \gamma \bar{a}_{3} a_{1} a_{4}-i \nu a_{2}\left|a_{2}\right|^{\beta}, \\
i \dot{a}_{3} & =2 \gamma \bar{a}_{2} a_{1} a_{4}-i \nu a_{3}\left|a_{3}\right|^{\beta}, \\
i \dot{a}_{4} & =2 \gamma \bar{a}_{1} a_{2} a_{3}+\sigma \dot{W}_{4}(t),
\end{aligned}
$$

where $\dot{W}_{1}$ and $\dot{W}_{4}$ are white noise. One reason for using white-noise forcing is the following: if we were to use deterministic forcing, the first oscillator could reach equilibrium with the forcing by a frequency detuning, and no more energy would be added to the system. This is not possible with stochastic forcing, and in the case of white noise the amount of energy entered into the system is exactly controllable.

Once we add white-noise forcing, we must damp the system somewhere, or the energy would diverge. Furthermore, once we force one mode (say $a_{1}$ ) then we must force $a_{4}$ with white noise, and of the same amplitude, to hope to have a statistical steady-state. (Otherwise, the Manley-Rowe relations would imply that $\left\langle\left|a_{1}\right|^{2}-\left|a_{4}\right|^{2}\right\rangle$ would diverge.) There is no reason other than symmetry to choose the damping strengths to be equal, but we do that here and exploit this symmetry.

Now, consider $(2.5)$ with $W_{1}=W_{4}$ and initial data so that $a_{1}(0)=$ $a_{4}(0)$, and $a_{2}(0)=a_{3}(0)$. Then

$$
a_{1}(t)=a_{4}(t), \quad a_{2}(t)=a_{3}(t), \text { for all } t,
$$

and (2.5) reduces to (2.1). The duet system is not a model for two interacting modes in a PDE such as (2.2), but it represents the reduction of quartet dynamics to an invariant submanifold.

\subsection{Relation to wave breaking}

(2.1) with nonlinear damping $(\beta \neq 0)$ is also reminiscent of wave breaking. In particular, wave breaking is naturally parameterized through a nonlinear dissipation of the form proposed above, with $\beta=1$. To see this, consider the prototypical model for wave breaking, i.e. the inviscid Burgers equation

$$
u_{t}+\left(\frac{1}{2} u^{2}\right)_{x}=0 .
$$


Here the energy $e=\frac{1}{2} u^{2}$ satisfies

$$
e_{t}+\left(\frac{2 \sqrt{2}}{3} e^{3 / 2}\right)_{x}=0 .
$$

The energy dissipation integrated over space is proportional to the sum of the jumps of $e^{3 / 2}$ at shocks. In a profile of fixed shape - say a sawtooth for the asymptotic behavior of Burgers in a periodic domain- which can be parameterized by the amplitude $a$ of its first Fourier mode, the energy $e$ is proportional to $|a|^{2}$, while the dissipation rate $e_{t}$ is proportional to $|a|^{3}$. This is the result of our nonlinear dissipation when the parameter $\beta$ is set to one.

\section{Elementary properties of the duet system}

The system (2.1) is Hamiltonian with

$$
H=\gamma\left(a_{1}^{2} \bar{a}_{2}^{2}+\bar{a}_{1}^{2} a_{2}^{2}\right),
$$

and the mass, momentum, and linear energy in (2.4) all become the same quantity, which we will now call the energy, denoted

$$
E=\left|a_{1}\right|^{2}+\left|a_{2}\right|^{2} \text {. }
$$

It will be convenient to use the variables $a_{k}=\rho_{k} e^{i \theta_{k}}, \xi_{k}=\rho_{k}^{2}$, giving

$$
\left\{\begin{array}{l}
d \xi_{1}=\left(4 \gamma \xi_{1} \xi_{2} \sin (2 \theta)+\sigma^{2}\right) d t+\sigma \sqrt{2 \xi_{1}} d W_{1} \\
d \xi_{2}=\left(-4 \gamma \xi_{1} \xi_{2} \sin (2 \theta)-2 \nu \xi_{2}^{\beta / 2+1}\right) d t \\
d \theta=2 \gamma\left(\xi_{2}-\xi_{1}\right) \cos (2 \theta) d t+\frac{\sigma}{\sqrt{2 \xi_{1}}} d W_{\theta}
\end{array}\right.
$$

where $W_{1}$ and $W_{\theta}$ are independent white noises. It is straightforward to see that for any fixed $\beta$ this system has a single non-dimensional parameter, which we define here as

$$
D=\gamma^{-1} \sigma^{2 \frac{\beta-2}{\beta+2}} \nu^{\frac{4}{\beta+2}}
$$

Now, in the interests of deciding which scaling limits are appropriate below, we will formally compute the expected value of the size of the two amplitudes $\xi_{1}, \xi_{2}$.

First, we calculate

$$
\frac{d}{d t}\left\langle\xi_{1}\right\rangle=4 \gamma\left\langle\xi_{1} \xi_{2} \sin (2 \theta)\right\rangle+\sigma^{2}
$$




$$
\frac{d}{d t}\left\langle\xi_{2}\right\rangle=-4 \gamma\left\langle\xi_{1} \xi_{2} \sin (2 \theta)\right\rangle-2 \nu\left\langle\xi_{2}^{\beta / 2+1}\right\rangle
$$

where $\langle\cdot\rangle$ denotes the expectation with respect to the noise. Adding these gives

$$
\frac{d}{d t}\langle E\rangle=\sigma^{2}-2 \nu\left\langle\xi_{2}^{\beta / 2+1}\right\rangle
$$

and thus if there is a steady-state, it must be true that

$$
\left\langle\xi_{2}^{\beta / 2+1}\right\rangle=\frac{\sigma^{2}}{2 \nu}
$$

For the case of linear damping $(\beta=0)$ this gives an exact bound for the average energy of $a_{2}$. If $\beta>0$, then this is an upper bound, as using Jensen's inequality gives

$$
\left\langle\xi_{2}\right\rangle \leq\left\langle\xi_{2}^{\beta / 2+1}\right\rangle^{\frac{2}{\beta+2}}=\left(\frac{\sigma^{2}}{2 \nu}\right)^{\frac{2}{\beta+2}} .
$$

On the other hand, we can also consider the equation

$$
d \ln \xi_{2}=-4 \gamma \xi_{1} \sin (2 \theta)-2 \nu \xi_{2}^{\beta / 2}
$$

After averaging, and again assuming the existence of a steady-state, we obtain

$$
\left\langle\xi_{1} \sin (2 \theta)\right\rangle=\frac{-\nu}{2 \gamma}\left\langle\xi_{2}^{\beta / 2}\right\rangle,
$$

and using the fact that $\left\langle\xi_{1}\right\rangle \geq\left|\left\langle\xi_{1} \sin (2 \theta)\right\rangle\right|$, we have

$$
\left\langle\xi_{1}\right\rangle \geq \frac{\nu}{2 \gamma}\left\langle\xi_{2}^{\beta / 2}\right\rangle \text {. }
$$

\section{Numerics and predictions}

We used the standard first-order Euler scheme throughout to simulate the equations here. In various cases, we sometimes simulated the original equations (3.2), and at other times different scaled versions of the equations, for example (6.1) were typically used for large $D$ values, and (5.1) were typically used for small $D$ values. We will state in each case below which equations we simulated. Furthermore, in all numerical simulations for $\beta=0$, we actually simulated $\ln \xi_{2}$ instead of $\xi_{2}$. As we will see from the numerics and analysis in this paper, in this $\beta=0$ regime, $\xi_{2}$ tends to become very close to 0 : in fact, $\ln \left(1 / \xi_{2}\right)$ reaches $O(D)$ frequently during many realizations.

We will analytically explain all of the observations of this section in Sections 5 and 6 below. 


\subsection{Scaling of amplitudes of $\xi_{1}, \xi_{2}$ with $D$}

Figures 4.1 and 4.2 show a series of of experiments concerning which of the bounds (3.3),(3.4) are saturated in which parameter regimes. Figure 4.1 corresponds the system (3.2) with $\beta=0, \sigma=\gamma=1$, and various $\nu$ in the range 0.2 to 8.0 . For these choices of parameters, $D=\nu^{2}$, so we are plotting $D$ values from 0.04 to 64.0 . To create this graph, we simulated between 10 and 25 realizations of the system from $t=0$ to $t=10^{5}$, and we calculated the time and ensemble average starting at $t=10^{4}$ of $\left\langle\xi_{1}\right\rangle$ and $\left\langle\xi_{2}\right\rangle$. We took timesteps ranging from $\Delta t=10^{-3}$ to $\Delta t=10^{-5}$. As we will see from the analysis below, the system becomes stiff for some values of $D$ and we took smaller timesteps and larger ensembles when appropriate. We have plotted $\left\langle\xi_{1}\right\rangle$ with circles and $\left\langle\xi_{2}\right\rangle$ with stars, and plotted the bounds (3.3) and (3.4) with solid lines. The bounds (3.3),(3.4) hold, and furthermore (3.3) is exact as predicted. Also, the inequality (3.4) seems to get closer to an equality as $D$ gets larger. Furthermore, as $D$ becomes small, the amplitudes $\xi_{1}$ and $\xi_{2}$ approach each other.

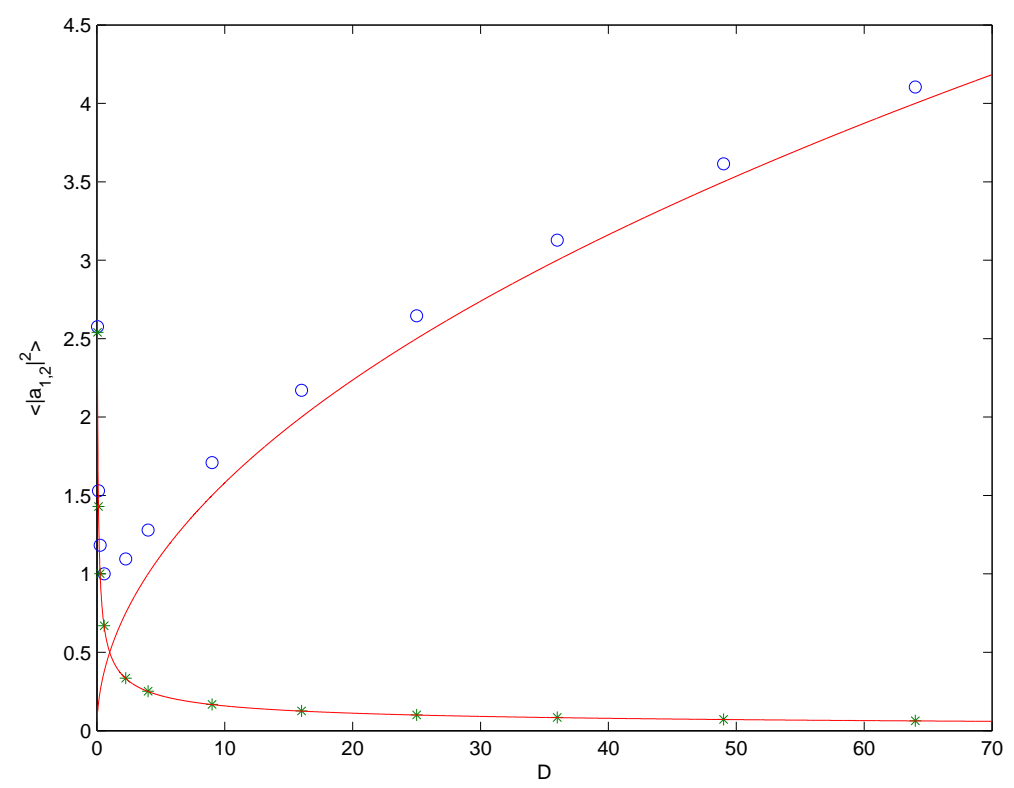

Figure 4.1. $\left\langle\xi_{1}\right\rangle$ (circles) and $\left\langle\xi_{2}\right\rangle$ (asterisks) as functions of $D=\nu^{2} / \sigma^{2} \gamma$ for $\beta=0$, with (3.4),(3.3) plotted as solid lines.

Figure 4.2 corresponds to the system with $\beta=5, \sigma=\gamma=1$, and 


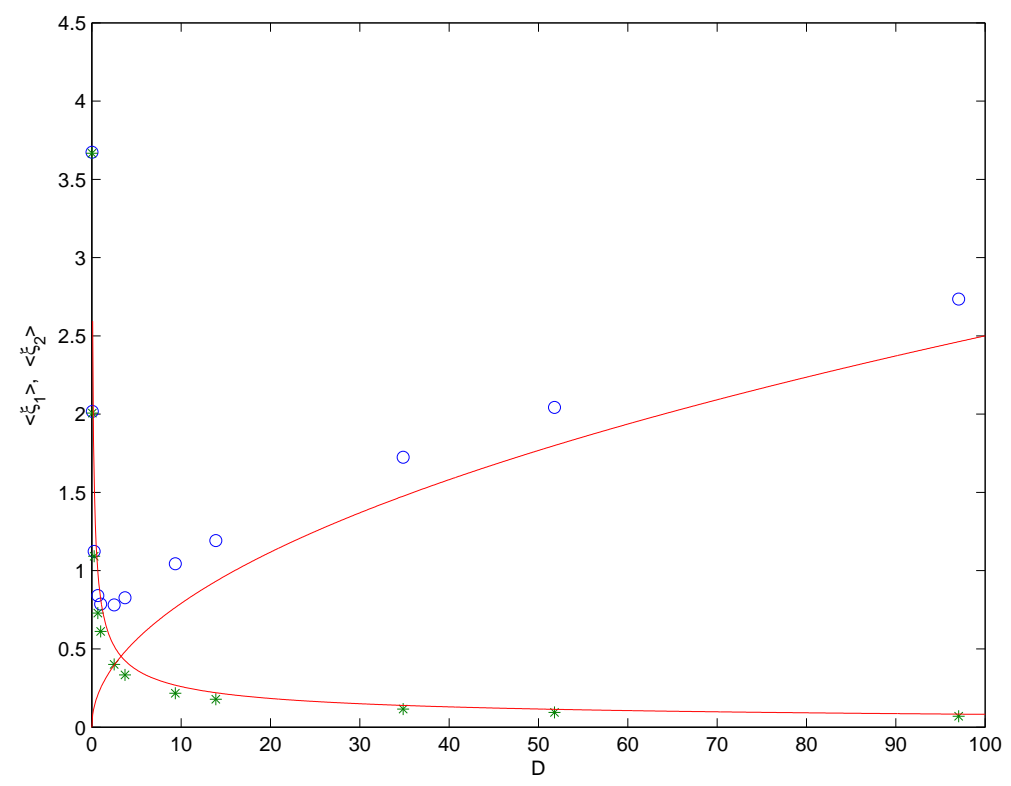

Figure 4.2. $\left\langle\xi_{1}\right\rangle$ (circles) and $\left\langle\xi_{2}\right\rangle$ (asterisks) as functions of $D=$ $\gamma^{-1} \sigma^{6 / 7} \nu^{4 / 7}$ for $\beta=5$, with (3.3),(3.4) plotted as solid lines. Note that for $\beta \neq 0$, we need to calculate $\left\langle\xi_{2}\right\rangle$ before we can draw the curve which is the lower bound for $\left\langle\xi_{1}\right\rangle$.

various $\nu$ in the range 0.1 to 3000 . In this case we simulated the equations directly with ensemble sizes, timesteps, and time domains similar to the $\beta=0$ case. For these choices of parameters, $D=\nu^{4 / 7}$, so we are plotting $D$ values from near $1 / 4$ to near 100. Again, one sees that the bounds (3.3),(3.4) hold. However, notice that the bounds are not as sharp in this case. Again, for small $D$, the amplitudes are nearly equal.

\subsection{Pathwise dynamics}

In all of the following numerical simulations, we simulated rescaled versions of the equations ((6.1) for large $D$, and (5.1) for small $D)$. Again, for $\beta=0$ we also simulated $\ln \xi_{2}$ instead of $\xi_{2}$ directly.

Figure 4.3 shows realizations of the system for various $D$ and $\beta$. As one can see from the two pictures in the bottom row, for small $D$ the two modes oscillate around each other in much the same way as in the unforced system. For large $D$, however, the scenario is quite different, and even depends sensitively on the value of $\beta$. In the case of large $D$ 

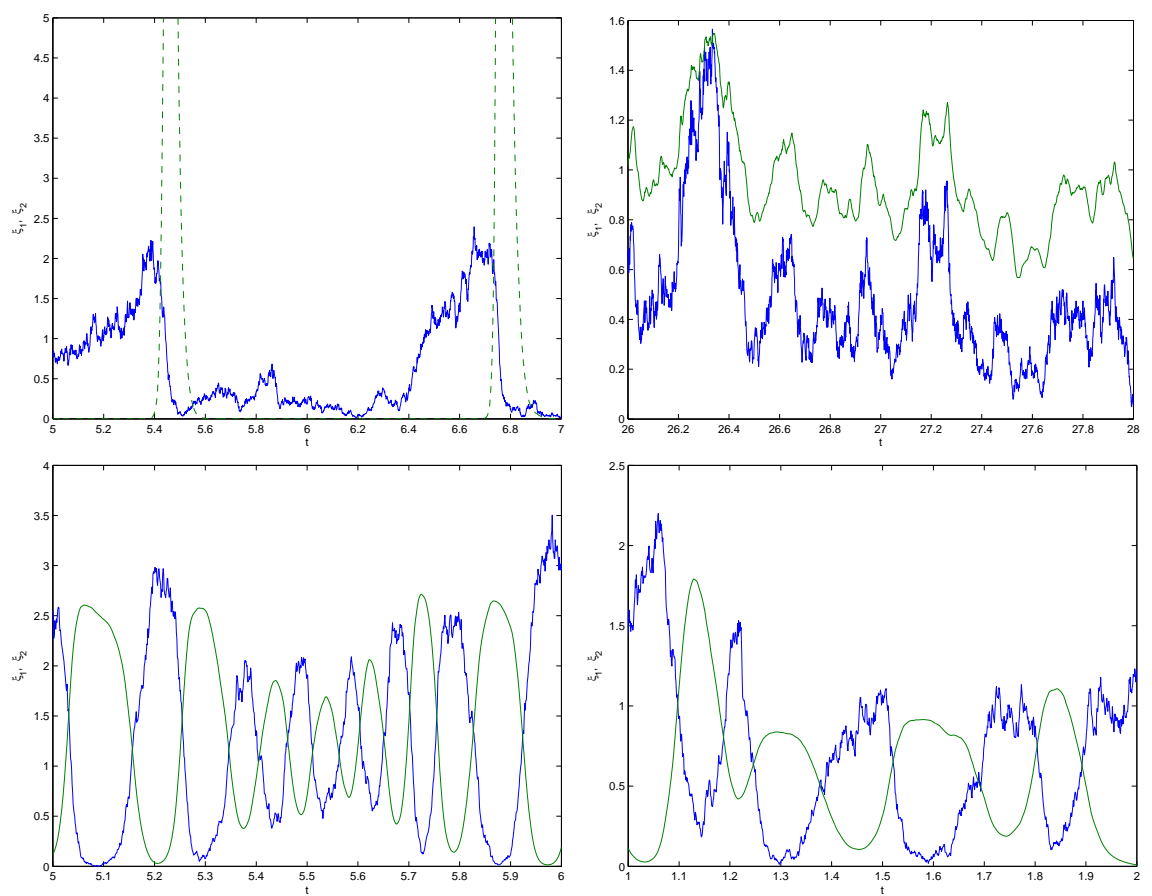

Figure 4.3. Realizations of (6.1) for various values of $D$ and $\beta$. In each case, the blue curve is $\xi_{1}$ and the green curve is $\xi_{2}$. The left column correspond to $\beta=0$ while the right column corresponds to $\beta=5$, and the first row correspond to $D=25$ while the bottom row corresponds to $D=0.1$.

but linear damping, the second oscillator stays pinned to 0 for most of the time, undergoing brief outbursts intermittently. On the other hand, for large $D$ and $\beta>0$, the amplitudes of $a_{1}$ and $a_{2}$ are locked.

\subsection{Equilibrium probability densities}

Figure 4.4 show the (numerically-calculated) densities of $\xi_{1}, \xi_{2}$ for various values of $D$ and $\beta$. For small $D$, one sees that the two oscillators are quite similar for either choice of $\beta$. There is one difference, in that for $\beta=0$, the measures are indistinguishable from Gaussian, while for $\beta>0$, the measures are definitely non-Gaussian. For large $D$, the two oscillators act quite differently. We see that for the case of $\beta=0, \xi_{1}$ is close to Gaussian, but the measure for $\xi_{2}$ is quite different, having a long tail. For the case 

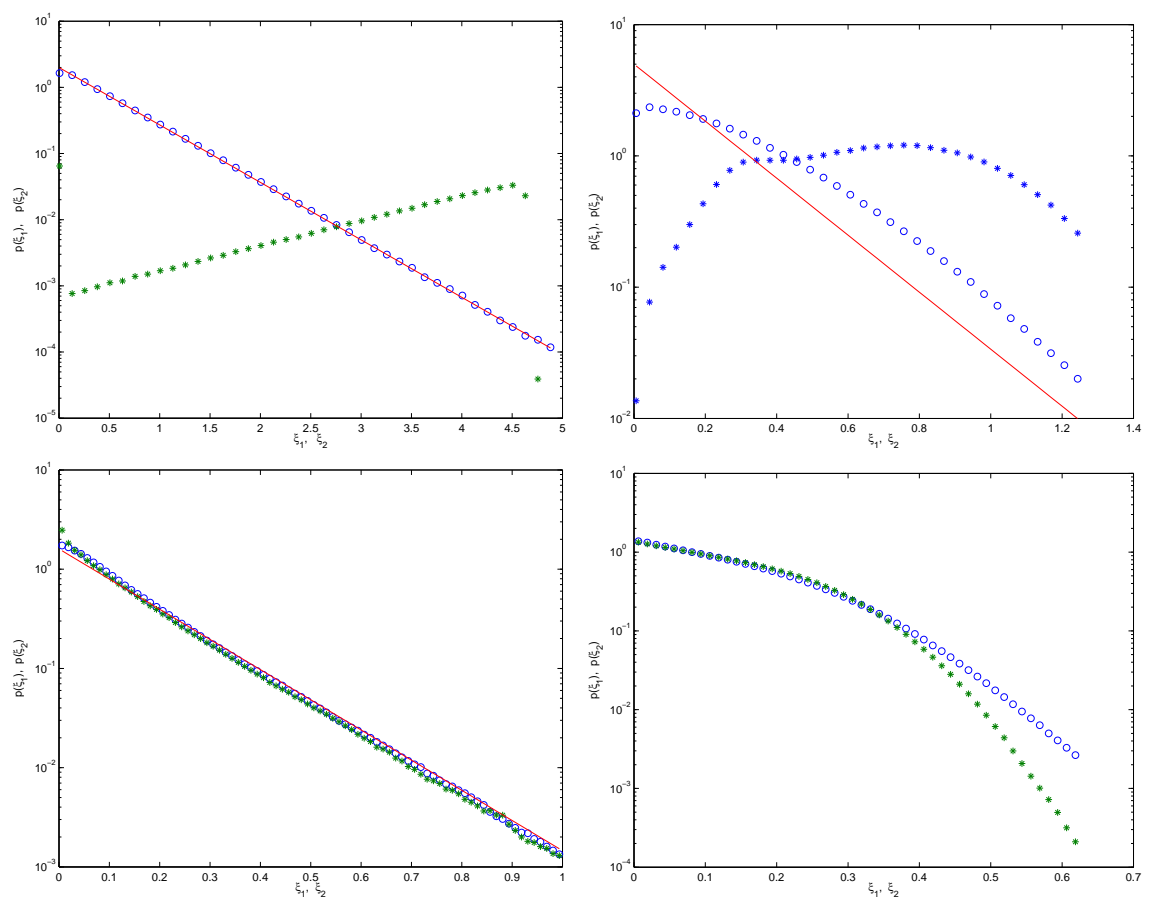

Figure 4.4. Numerically-computed densities of (6.1) for various values of $D$ and $\beta$. The left column correspond to $\beta=0$ while the right column corresponds to $\beta=5$, and the first row correspond to $D=25$ while the bottom row corresponds to $D=0.1$. Where the graph includes a descending line, this is a graph of an exponential.

of $\beta>0$, we see that neither measure is Gaussian.

\section{$5 \quad D \ll 1-$ The quasi-equilibrium regime}

Combining (3.3) with the numerical observation (see figures 4.1, 4.2) that the average energy of $a_{1}$ and $a_{2}$ are the same for small $D$ suggests the scalings

$$
\begin{aligned}
\xi_{j} & =\sigma^{\frac{4}{\beta+2}} \nu^{-\frac{2}{\beta+2}} \tilde{\xi}_{j}, \\
t & =\sigma^{-\frac{2 \beta}{\beta+2}} \nu^{-\frac{2}{\beta+2}} \tilde{t} .
\end{aligned}
$$


This scaling can be obtained by assuming that (3.3) is saturated and that the $\xi_{j}$ are of the same size. This gives us

$$
\left\{\begin{array}{l}
d \xi_{1}=4 D^{-1} \xi_{1} \xi_{2} \sin (2 \theta) d t+1 d t+\sqrt{2 \xi_{1}} d W_{1} \\
d \xi_{2}=-4 D^{-1} \xi_{1} \xi_{2} \sin (2 \theta) d t-2 \xi_{2}^{\frac{\beta+2}{2}} d t \\
d \theta=2 D^{-1}\left(\xi_{2}-\xi_{1}\right) \cos (2 \theta) d t+\frac{1}{\sqrt{2 \xi_{1}}} d W_{\theta}
\end{array}\right.
$$

where we have dropped tildes. Also, notice that every term representing Hamiltonian dynamics has a $D^{-1}$ in front of it, and all of the terms arising from forcing and damping are of order 1.

What we show below is that there exist functions $\tilde{T}_{\alpha}, \psi$ (see (A.6) for definitions) so that the dynamics of (5.1) can be approximated by

$$
\left\{\begin{array}{l}
d E=2\left(1-E^{\beta / 2+1} \tilde{T}_{\beta / 2+1}(h)\right) d t+\sqrt{E} d W_{1}+\sqrt{h^{2} / E} d W_{2}, \\
d h=-2 E^{\beta / 2} h \tilde{T}_{\beta / 2}(h) d t+\sqrt{(4 \psi(h)-h) h / E} d W_{2},
\end{array}\right.
$$

where $h=2 H / E^{2}$. In particular, $\tilde{T}_{0}=2$ and $\tilde{T}_{1}=1$, so if $\beta=0$, we get the simpler SDE

$$
\begin{aligned}
d E & =2(1-E) d t+\sqrt{E} d W_{1}+\sqrt{h^{2} / E} d W_{2}, \\
d h & =-4 h d t+\sqrt{(4 \psi(h)-h) h / E} d W_{2} .
\end{aligned}
$$

This represents a dimensional reduction of the original system. We have only reduced the original system (2.1) by two degrees of freedom, but this formulation has the advantage that it has no small parameter. In the $D \ll 1$ limit, (5.1) are a stiff set of equations. From a numerical point of view, this technique would represent a clear savings in the small $D$ limit. This viewpoint is entirely analogous to the reduction to the slow manifold in singular perturbation theory for deterministic ODE.

\subsection{Consequences of (5.2)}

Converting (5.2) to the appropriate Fokker-Planck equation gives

$$
\begin{gathered}
F_{t}=\left(2\left(E^{\beta / 2+1} \tilde{T}_{\beta / 2+1}(h)-1\right) F\right)_{E}+\left(E^{\beta / 2} h \tilde{T}_{\beta / 2}(h) F\right)_{h} \\
+(E F)_{E E}+(2 h F)_{E h}+\left(4 h E^{-1} \psi(h) F\right)_{h h .}
\end{gathered}
$$

The steady-state distribution is any $F$ which satisfies

$$
\begin{gathered}
0=\left(2\left(E^{\beta / 2+1} \tilde{T}_{\beta / 2+1}(h)-1\right) F\right)_{E}+\left(E^{\beta / 2} h \tilde{T}_{\beta / 2}(h) F\right)_{h} \\
+(E F)_{E E}+(2 h F)_{E h}+\left(4 h E^{-1} \psi(h) F\right)_{h h} .
\end{gathered}
$$


on the domain $0 \leq E \leq \infty,-1 \leq h \leq 1$, with Dirichlet boundary conditions.

In certain cases, we can solve (5.4) exactly. We first simplify by calculating the marginal distribution in $E$ and $h$. We integrate (5.4) in $h$, obtaining

$$
2\left(C_{\beta / 2+1} E^{\beta / 2+1}-1\right) \tilde{F}_{E}+(E \tilde{F})_{E E}=0
$$

where

$$
\tilde{F}=\int_{-1}^{1} d h F(E, h), \quad C_{\alpha}=\int_{-1}^{1} d h \tilde{T}_{\alpha}(h) .
$$

The only solution of (5.5) which is bounded at infinity is

$$
\tilde{F}(E)=E \exp \left(-k E^{1+\beta / 2}\right)
$$

with $k=4 C_{\beta / 2+1} /(\beta+2)$. We note that this agrees with the results obtained in [9] and [10], where a similar analysis was done using the implicit assumption that the distribution only depends upon $E$. Also, one can see from figure 5.1 that this agrees quite well, for various choices of $\beta$, with the numerically calculated distribution. Note that this distribution is not Gaussian for $\beta>0$.

Furthermore, in the case where $\beta=0$, it turns out that the solution for $F$ in (5.3) actually separates, and the PDE can be solved explicitly to obtain

$$
F(E, h)=E e^{-E} \frac{\exp (-\chi(h) / 2)}{h \psi(h)},
$$

where $\chi(h)=\int_{0}^{h} d h^{\prime} \psi\left(h^{\prime}\right)$. The authors cannot find such a nice separation of variables for the $\beta>0$ case.

\subsection{Derivation of (5.2) and (5.3)}

The corresponding generator of the diffusion in (5.1) is

$$
\frac{1}{D} L_{H}+L_{F D}
$$

where

$$
\begin{aligned}
L_{H} & =4 \xi_{1} \xi_{2} \sin (2 \theta)\left(\partial_{\xi_{1}}-\partial_{\xi_{2}}\right)+2\left(\xi_{2}-\xi_{1}\right) \cos (2 \theta) \partial_{\theta} \\
L_{F D} & =\partial_{\xi_{1}}+\xi_{1} \partial_{\xi_{1} \xi_{1}}^{2}-2 \xi_{2}^{\frac{\beta+2}{2}} \partial_{\xi_{2}}+\frac{1}{4 \xi_{1}} \partial_{\theta}^{2}
\end{aligned}
$$



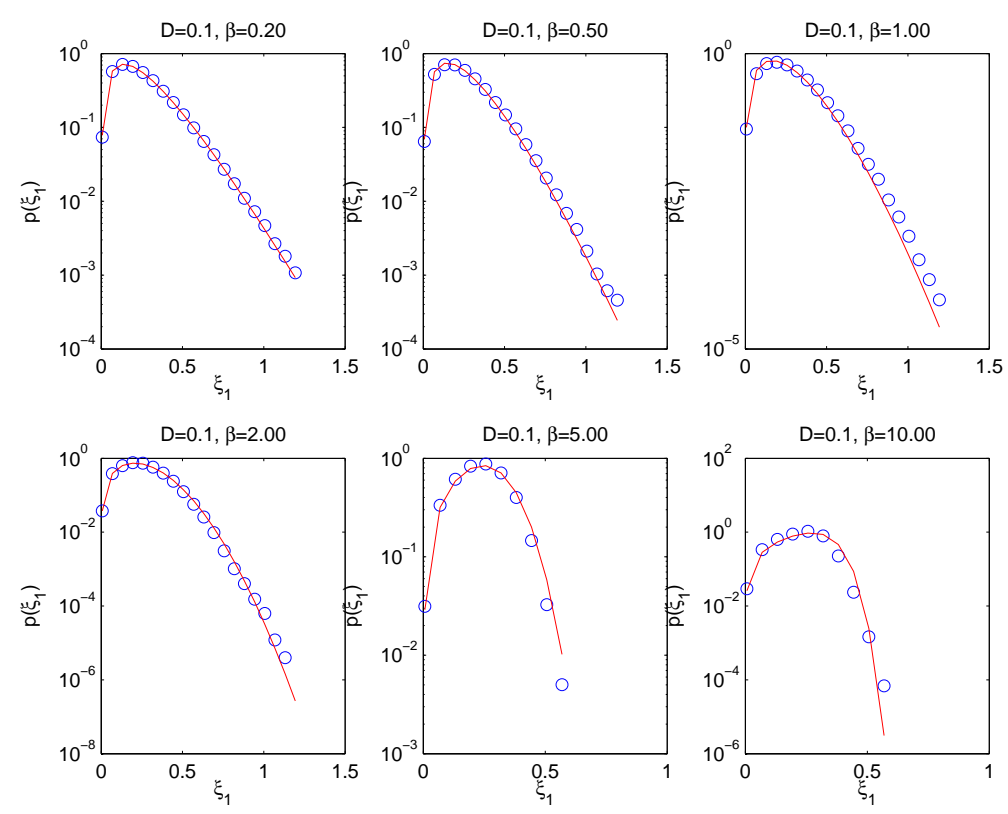

Figure 5.1. A pdf of the energy of (6.1) for small $D$ and various values of $\beta$. The circles are the numerically determined distributions, and the line is the prediction made in (5.6), with $k$ determined numerically.

We are considering $D \ll 1$, so that the $L_{H}$ term dominates. First note that $L_{H}$ is skew. This is because it is written in the form $L_{H}=F \cdot \nabla$, where $F$ is divergence-free, and thus $L_{H}^{*}=-(F \cdot \nabla)+\operatorname{div} F=-L_{H}$.

By inspection, one can see that the null space of $L_{H}$ (and also $L_{H}^{*}$ for that matter) is made up of functions of $H$ and $E$. This makes sense, since it says exactly that any positive function of the conserved quantities can be used to construct an invariant measure for the unforced system.

We write

$$
\partial_{t} f-L^{*} f=\partial_{t} f-\frac{1}{D} L_{H}^{*} f-L_{F D}^{*} f=0 .
$$

Let us assume that $f$ can be developed in a series as

$$
f=f_{0}+D f_{1}+D^{2} f_{2}+\cdots
$$

and plugging this into (5.9) gives

$$
\begin{aligned}
& L_{H}^{*} f_{0}=0, \\
& L_{H}^{*} f_{1}=\partial_{t} f_{0}-L_{F D}^{*} f_{0} .
\end{aligned}
$$


The first term tells us that $f_{0}$ is a function of $H$ and $E$ and we will denote this $F(H, E)$. The second equation gives a compatibility condition of the form

$$
\partial_{t} F-L_{F D}^{*} F \in \mathcal{R}\left(L_{H}^{*}\right)=\mathcal{N}\left(L_{H}\right)^{\perp} .
$$

This means that for any function $G \in \mathcal{N}\left(L_{H}\right)$ (i.e. any function $G=$ $G(H, E))$, we must have

$$
\int d \xi_{1} d \xi_{2} d \theta\left(\partial_{t} F+L_{F D}^{*} F\right) G=0
$$

We can integrate by parts to write this as

$$
\int F_{t} G+F L_{F D} G=0
$$

where, again, $G$ is any test function of $E$ and $H$. We stress that we are not interested in determining $f_{1}$ or any other term in the asymptotic expansion; we are only trying to determine the form of $f_{0}$. What we will do in (5.10) is rewrite the $F L_{F D} G$ integral so that it is in $H$ and $E$ coordinates, then average over the remaining coordinate to obtain an operator which depends solely on $H$ and $E$. The way we do this is first add two integrals and delta functions, as in

$$
\begin{aligned}
& \int_{0}^{\infty} d \hat{E} \int_{-\hat{E}^{2} / 2}^{\hat{E}^{2} / 2} d \hat{H} \int_{0}^{2 \pi} d \theta \int_{0}^{\infty} d \xi_{1} \int_{0}^{\infty} d \xi_{2} \\
& \delta\left(H\left(\xi_{1}, \xi_{2}, \theta\right)-\hat{H}\right) \delta\left(E\left(\xi_{1}, \xi_{2}, \theta\right)-\hat{E}\right) F(H, E) L_{F D}\left(H, E, \xi_{1}, \xi_{2}, \theta\right) .
\end{aligned}
$$

We use the two delta functions to remove two $d \xi$ integrals, and then do the $d \theta$ integral explicitly (see Appendix A for details) to obtain

$$
\int_{0}^{\infty} d \hat{E} \int_{-\hat{E}^{2} / 2}^{\hat{E}^{2} / 2} d \hat{H} J(\hat{H}, \hat{E}) F(\hat{H}, \hat{E}) \tilde{\phi}(\hat{H}, \hat{E}),
$$

where

$$
\begin{aligned}
J(\hat{H}, \hat{E})= & \frac{2}{\hat{E} \sqrt{1+a}} K\left(\sqrt{\frac{1-a}{1+a}}\right) \\
\tilde{\phi}(\hat{H}, \hat{E})= & \left(2-2 T_{\beta / 2+1}(\hat{H}, \hat{E})\right) G_{E}-\hat{H} T_{\beta / 2}(\hat{H}, \hat{E}) G_{H} \\
& +\hat{E} G_{E E}+2 \hat{H} G_{H E}+4 \hat{E} \hat{H} \psi(\hat{H}, \hat{E}) G_{H H},
\end{aligned}
$$


with $a=\left|2 \hat{H} / \hat{E}^{2}\right|, K$ is the elliptic $K$-function, and $T_{\alpha}$ and $\psi$ are defined below in (A.6). Although the expression $T_{\alpha}$ is rather complex, it is worth noting here that $T_{0}=2$ and $T_{1}=\hat{E}$, so that for $\beta=0$ the expression for $\tilde{\phi}(\hat{H}, \hat{E})$ is much nicer, namely

$$
2(1-\hat{E}) G_{E}-2 \hat{H} G_{H}+\hat{E} G_{E E}+2 \hat{H} G_{E H}+4 \hat{E} \hat{H} \psi G_{H H} .
$$

Since $J$ really represents the Jacobian of changing variables to $H, E$, we want to understand the evolution of $X=J F$. We plug (5.12) into (5.10), integrate by parts, and note that $G$ is arbitrary, to obtain

$$
\begin{aligned}
X_{t}=\left(2\left(T_{\beta / 2+1}-1\right) X\right)_{E}+\left(H T_{\beta / 2} X\right)_{H} \\
+(E X)_{E E}+(2 H X)_{E H}+(2 E H \psi X)_{H H} .
\end{aligned}
$$

Further making the change $h=2 H / E^{2}$ and writing $F$ for $X$ gives (5.3). This is of course equivalent to the SDE in (5.2).

\section{$6 \quad D \gg 1$, The non-equilibrium regime}

In contrast to the small $D$ asymptotics of section 5 , we will see here that there is a vast qualitative difference between the case where $\beta=0$ and where $\beta>0$, and we will deal with these two cases separately below. In both cases, however, we will do the scaling

$$
\xi_{1}=\gamma^{-1} \sigma^{\frac{2 \beta}{\beta+2}} \nu^{\frac{2}{\beta+2}} \tilde{\xi}_{1}, \quad \xi_{2}=\sigma^{\frac{4}{\beta+2}} \nu^{-\frac{2}{\beta+2}} \tilde{\xi}_{2}, \quad t=\gamma^{-1} \sigma^{-\frac{4}{\beta+2}} \nu^{\frac{2}{\beta+2}} \tilde{t} .
$$

This is obtained by assuming that the bounds (3.3) and (3.4) are saturated. The scaling gives us (after dropping tildes)

$$
\left\{\begin{array}{l}
d \xi_{1}=4 \xi_{1} \xi_{2} \sin (2 \theta) d t+d t+\sqrt{2 \xi_{1}} d W_{1} \\
d \xi_{2}=D\left(-4 \xi_{1} \xi_{2} \sin (2 \theta)-2 \xi_{2}^{\beta / 2+1}\right) d t \\
d \theta=2\left(\xi_{2}-D \xi_{1}\right) \cos (2 \theta) d t+\frac{1}{\sqrt{2 \xi_{1}}} d W_{\theta}
\end{array}\right.
$$

\subsection{Nonlinear damping, $\beta>0$}

The $\beta>0$ case turns out to be the more straightforward of the two cases. Let us rewrite the equation for $\xi_{2}$ in (6.1) as

$$
d \xi_{2}=-2 D \xi_{2}\left(2 \sin (2 \theta) \xi_{1}+\xi_{2}^{\beta / 2}\right)
$$

We write $\alpha(t)=\sin (2 \theta)$, and for now think of this as a control parameter which varies in time in some specified way. For any fixed $\alpha$, this equation 
is strongly stable to the curve $\xi_{2}=\left(-2 \alpha \xi_{1}\right)^{2 / \beta}$ (by strongly stable we mean it has a large negative eigenvalue). Furthermore, if $\xi_{2} \ll D \xi_{1}$, the equation for $\theta$ in (6.1) is strongly stable to the value of $\theta$ for which $\cos (2 \theta)=0$ and $\sin (2 \theta)=-1$, namely $\theta=3 \pi / 4$.

If we happen to choose an initial condition with $\xi_{2}=O(D)$, then the dynamics of $\xi_{2}$ will pull the solution rapidly into a neighborhood of the manifold

$$
\xi_{2}=\left(-2 \alpha \xi_{1}\right)^{2 / \beta}
$$

Once we are there, the value of $\theta$ locks to $\theta=3 \pi / 4$ and thus $\alpha$ locks to -1 , and this causes $\xi_{2}$ to lock to the manifold $\xi_{2}=\left(2 \xi_{1}\right)^{2 / \beta}$, and then $\xi_{2}$ stays $O(1)$.

We stress that although there are regions of phase space for which there is not phase- and amplitude-locking, this is a self-correcting phenomenon since the deterministic part of the vector field causes the system to leave these areas quickly. With the locking, the equation for $\xi_{1}$ in (6.1) becomes

$$
d \xi_{1}=\left(-2^{\frac{2 \beta+2}{\beta}} \xi_{1}^{1+\frac{2}{\beta}}+1\right) d t+\sqrt{2 \xi_{1}} d W
$$

and it is straightforward to calculate that the steady-state distribution for this equation is

$$
f\left(\xi_{1}\right)=C e^{-k \xi_{1}^{1+2 / \beta}}
$$

where in fact $k=2^{\frac{2 \beta+2}{\beta}} \beta /(\beta+2)$. Thus the steady-state distribution for the full system (6.1) is

$$
f\left(\xi_{1}\right) \delta\left(\xi_{2}-\left(2 \xi_{1}\right)^{2 / \beta}\right) \delta(\theta-3 \pi / 4)
$$

In figures 6.1 and 6.2 , we see that the numerics agree with these predictions. We mention that it is an implicit assumption in the above argument that $D$ be chosen sufficiently large, and the minimum such choice of $D$ is $\beta$-dependent. As $\beta \rightarrow 0$, one will need to choose larger values of $D$ to ensure phase- and amplitude-locking. It is an interesting observation that the distribution for $\xi_{1}$ is not Gaussian for any value of $\beta>0$.

\subsection{Linear damping, $\beta=0$}

The case $\beta=0$ is somewhat more complicated. If we consider (6.1) with $\beta=0$, we note that we might expect phase-locking of $\theta$ to occur, at least for most regions of the phase space, but now there is certainly no 

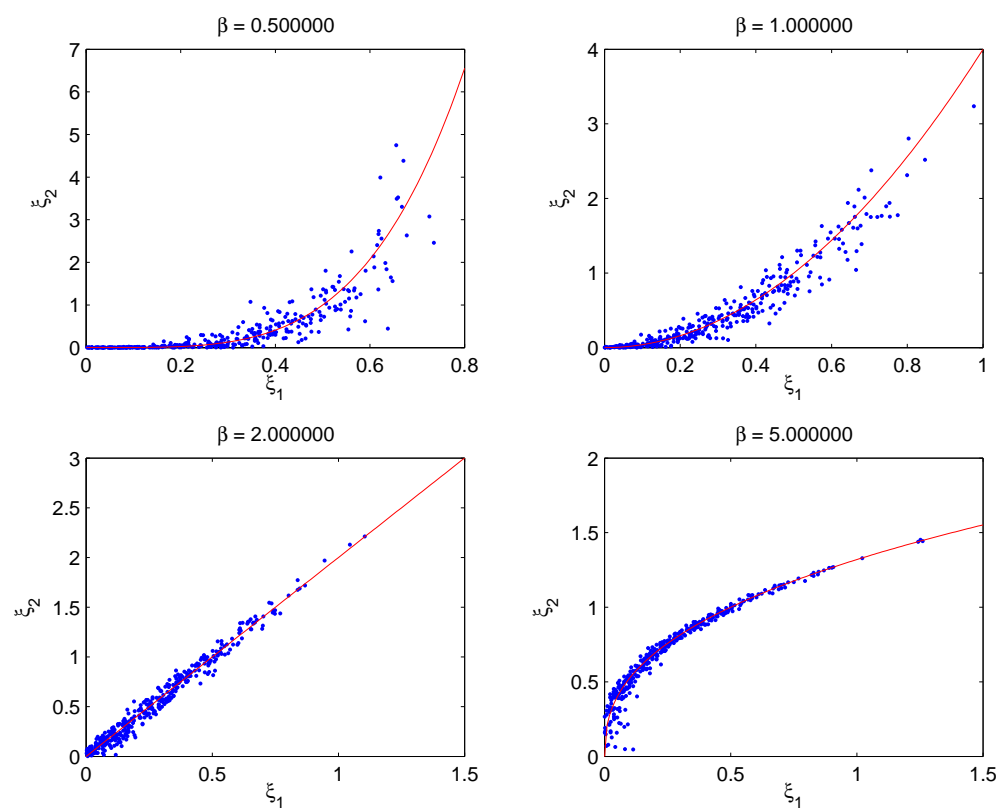

Figure 6.1. In each graph, the curve is $\xi_{2}=\left(2 \xi_{1}\right)^{2 / \beta}$ and the dots are the solution of $(6.1)$ at times $t=1000+25 k, D=250$.

mechanism which locks $\xi_{2}$ to $\xi_{1}$. The situation is further complicated because the $\theta$ does not stay locked for typical trajectories of the system.

We will show that the dynamics in the $\beta=0$ regime are of two phases which switch back and forth intermittently. The first phase is a quiescent one in which $\xi_{2}$ is pinned very near zero and $\xi_{1}$ undergoes a random walk. The second phase is characterized by a burst in the energy of $\xi_{2}$. We also will show that the quiescent phase is essentially unaffected in its duration and quality by $D$, although it is affected by noise, and the bursting phase is essentially unaffected by the noise, but is affected greatly by $D$. In fact, as we show below, the length, in time, of the bursts are $O(\ln D / D)$ and their amplitude is $O(D)$.

Let us consider the system where $\beta=0$, namely

$$
\left\{\begin{array}{l}
d \xi_{1}=\left(4 \sin (2 \theta) \xi_{1} \xi_{2}+1\right) d t+\sqrt{2 \xi_{1}} d W_{1} \\
d \xi_{2}=-2 D \xi_{2}\left(2 \sin (2 \theta) \xi_{1}+1\right) d t \\
d \theta=2\left(\xi_{2}-D \xi_{1}\right) \cos (2 \theta) d t+\left(\sqrt{2 \xi_{1}}\right)^{-1} d W_{\theta}
\end{array}\right.
$$

We will define the "quiescent" phase to be that in which $\xi_{2}<1$, and 

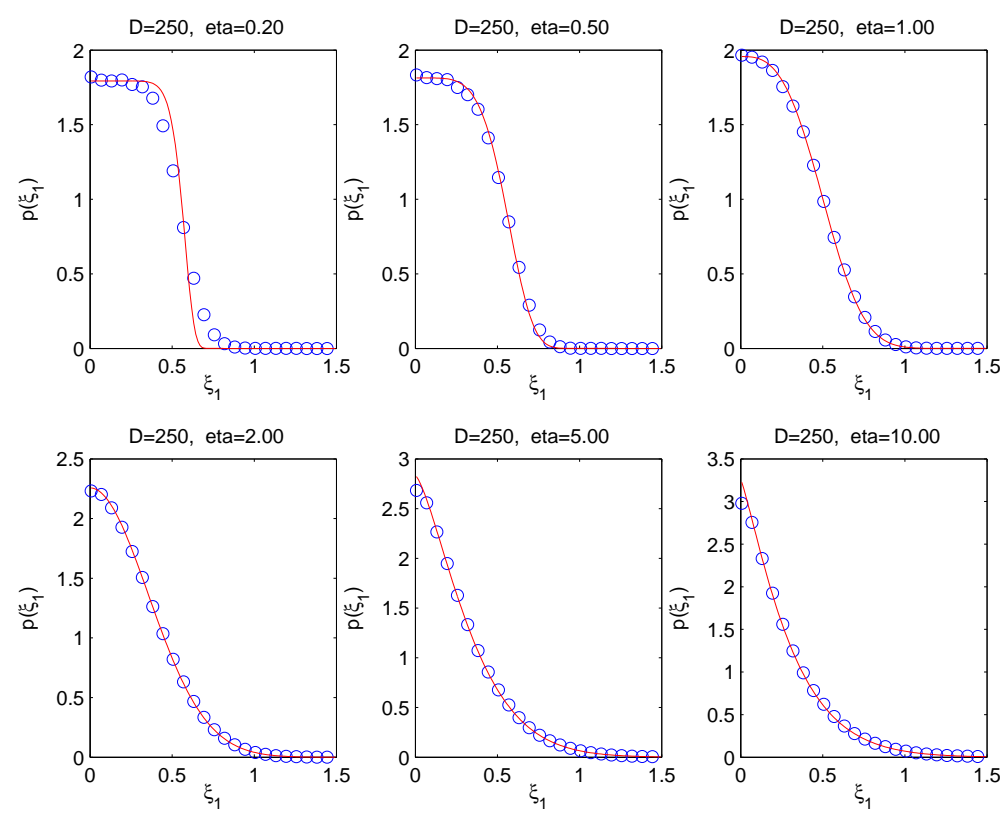

Figure 6.2. In each graph, the curve is the prediction given in (6.2) and the dots are the numerically computed distribution of (6.1).

the "bursting" phase to be that in which $\xi_{2}>1$. The 1 is arbitrary and chosen only for definiteness. When we are in the quiescent stage, it is easy to see that $\theta$ is locked to $\theta=3 \pi / 4$, and since it has a multiplier of $O(D)$, one can see that its variance is $O(1 / D)$ since $\xi_{1}$ is always of $O(1)$. In this phase we can simplify the system, using the substitution $\sin (2 \theta)=-1$, to obtain

$$
\begin{aligned}
& d \xi_{1}=\left(1-4 \xi_{1} \xi_{2}\right) d t+\sqrt{2 \xi_{1}} d W \\
& d \xi_{2}=2 D\left(2 \xi_{1}-1\right) \xi_{2} d t .
\end{aligned}
$$

The $\xi_{2}$ equation is linear, and we see by inspection that it always changing rapidly. It will be rapidly shrinking if $\xi_{1}<\frac{1}{2}$ and rapidly growing if $\xi_{1}>\frac{1}{2}$. Consider an initial condition $\left(\xi_{1}, \xi_{2}\right)=(A, 1)$ with $A<1 / 2$. Then $\xi_{2}$ moves toward zero rapidly. Effectively, the equation for $\xi_{1}$ does not then depend on $\xi_{2}$, and it is easy to see that it is a random walk with a positive drift.

If we think of $\xi_{1}(t)$ as a parameter forcing the equation for $\xi_{2}$ in (6.4), 
we get

$$
\xi_{2}=\exp \left[-2 D \int_{0}^{t} d s\left(2 \xi_{1}(s)-1\right)\right]
$$

In particular, the quiescent stage will last until the value of $t$ for which

$$
0=\int_{0}^{t} d s\left(2 \xi_{1}(s)-1\right) .
$$

This does not depend on $D$. In essence, the effect of large $D$ in the quiescent regime is to pin $\xi_{2}$ to 0 more strongly when $\xi_{1}<1 / 2$, and have it come back to 1 more quickly when $\xi_{1}>1 / 2$, but this effect cancels out, and $D$ will not affect the length of the quiescent stage. In particular, the mean value $\left\langle\xi_{1}\right\rangle$ takes when this phase ends is $1-A$, and this is a quantity independent of $D$.

In summary, as long as $D$ is sufficiently large, the length of time the quiescent phase lasts is independent of $D$, and the values that $\xi_{1}$ takes during this phase are also independent of $D$. On the other hand, the values that $\xi_{2}$ takes during this phase depend on $D$ greatly, in that they go like $e^{-D t}$ for the first half of this phase (while $\xi_{1}<1 / 2$ ) and then grow like $e^{D t}$ in the second half (while $\xi_{1}>1 / 2$ ).

Now we consider the bursting phase. We will see that the assumption that $\theta$ is locked in this phase is not valid. In fact, let us assume that $\theta$ is locked to $\theta=3 \pi / 4$, and show that this is not a self-consistent assumption. Consider the system (6.4). For definiteness, let us consider an initial condition $(B, 1)$ with $B>1 / 2$. It is easy to see that the bursting phase will not be greatly affected by the noise, since during the burst the deterministic vector field is everywhere $O(D)$. Thus we consider the deterministic equation

$$
\begin{aligned}
& d \xi_{1}=-4 \xi_{1} \xi_{2} d t \\
& d \xi_{2}=2 D\left(2 \xi_{1}-1\right) \xi_{2} d t
\end{aligned}
$$

and we consider the following problem. Given initial conditions $\left(\xi_{1}, \xi_{2}\right)=$ $(B, 1)$ with $B>1 / 2$, where and when does the trajectory "land", i.e. is it true that for some $t>0,\left(\xi_{1}(t), \xi_{2}(t)\right)=(C, 1)$, and what are $C$ and $t$ ? Noting that for this system, we have

$$
\frac{d \xi_{2}}{d \xi_{1}}=\frac{-D\left(2 \xi_{1}-1\right)}{2 \xi_{1}},
$$

we can integrate this to get

$$
\xi_{2}\left(\xi_{1}\right)=D \eta\left(\xi_{1}\right)+C:=D\left(\frac{\ln \left(\xi_{1}\right)}{2}-\xi_{1}\right)+C,
$$


and the solution which passes through $(B, 1)$ is

$$
\xi_{2}\left(\xi_{1}\right)=D\left(\eta\left(\xi_{1}\right)-\eta(B)\right)+1 .
$$

Since $\eta$ is a function which is concave down with maximum at $1 / 2$, this shows that $C<1 / 2$. Furthermore, this curve has maximum $O(D)$. This would seem to explain the bursting phenomenon well, since here we have a quick transition from $(B, 1)$ to $(C, 1)$ with an excursion height of $O(D)$, which brings us to the start of another quiescent phase.

Unfortunately, this argument is not quite correct, because one can see that the phase-locking assumption is not valid. Since the equation has a transition into the $\xi_{2}>D \xi_{1}$ region of phase-space (and in fact spends most of its time there), the locking in the $\theta$ equation switches signs, and $\theta$ now locks to $\pi / 4$. This changes the signs in the $d \xi_{j}$ equations so makes this analysis incorrect.

Now consider system (6.3), rescale $\xi_{1}=x, \xi_{2}=D y, \tau=D t$, and write $\alpha(t)=\sin (2 \theta)$. The equations become

$$
\begin{aligned}
& d x=4 \alpha x y+\frac{1}{D}, \\
& d y=-2 y(2 \alpha x+1), \\
& d \alpha=2(y-x)\left(1-\alpha^{2}\right),
\end{aligned}
$$

and our initial condition is now $\left(x_{0}, y_{0}\right)=(B, 1 / D)$. The quantity $\alpha$ wants to lock to either 1 or -1 depending on the values of $x$ and $y$. Let us split the positive quadrant into the two pieces $R_{1}=\left\{\xi_{1}>1 / 2\right\}$ and $R_{2}=\left\{\xi_{1}<1 / 2\right\}$. By a simple phase plane analysis, one sees that for $\alpha=-1$, the vector field points up and to the left in $R_{1}$ and down and to the right in $R_{2}$. On the other hand, if $\alpha=1$, then the vector field points down and to the right everywhere.

If we start at the point $(B, 1 / D)$, there are two possibilities of landing point (where we are "landing" at a point with $y=1 / D$ ). First, the trajectory can move up and to the left, cross the line $\xi_{1}=1 / 2$, and then land at some point to the left of $1 / 2$. This is the same picture we would obtain assuming $\theta=3 \pi / 4$ throughout the burst. Second, the trajectory can move to the right of the line $x=1 / 2$ above $y=1$, and then land to the right of $x=1 / 2$. But we will again be in the region where $\xi_{2} \ll D \xi_{1}$, and then the bursting process starts over again with this new initial condition. The second case is a sort of "multibump" bursting. In any case, the system will return quickly to the quiescent mode, no matter how many bumps it traverses. 
Furthermore, we claim that the multibump bursting is not important for large $D$ dynamics. Recall that the initial condition $B$, while random, has mean less than 1 . The probability of our having a burst which starts at, say, $\xi_{1}=10$ is quite rare but as one can see from figure 6.3 , for large $D$ the burst has to start at $\xi_{1}>10$ to get a multibump solution. Moreover, the probability of a multibump occurring goes to 0 as $D \rightarrow \infty$.

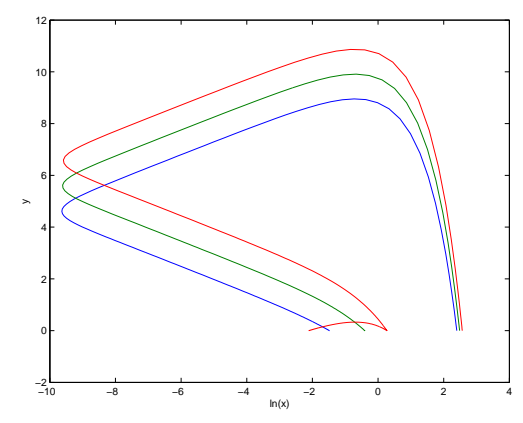

Figure 6.3. Evolution of system (6.5) for $D=10^{4}$ and three initial conditions: $(11,1 / D),(12,1 / D),(13,1 / D)$. The threshhold for getting a multibump solution seems to be somewhere between 12 and 13 .

We can calculate the length of time the burst takes directly from the vector field. Take a (non-multibump) burst starting at $(B, 1)$. Take $B^{*}=$ $(B+1 / 2) / 2$, and let $C^{*}$ be the solution to $\eta\left(B^{*}\right)=\eta\left(C^{*}\right)$. Then the solution will pass through the points $(B, 1),\left(B^{*}, O(D)\right),\left(C^{*}, O(D)\right)$, and $(C, 1)$. We can estimate how long it takes to pass though these points. To get from $(B, 1)$ to $\left(B^{*}, O(D)\right)$, the $\xi_{2}$ variable must move an $O(D)$ distance. In this region, $d \xi_{2}=O\left(D \xi_{2}\right)$, so that it goes like $e^{D t}$. It will thus take a $\ln (D) / D$ time to traverse this distance. The same argument holds for the transit from $C^{*}$ to $C$. Now, to get from $B^{*}$ to $C^{*}$, note that $d \xi_{1}=O\left(\xi_{1} \xi_{2}\right)=O\left(D \xi_{1}\right)$, and it has to move a $O(1)$ distance. This will take $O(1 / D)$ time. All in all, the burst will take $O(\ln (D) / D)$ time. Compare this with figure 6.4, where the circles represent the proportion of time the system spends in the bursting phase as a function of $D$. For this calculation, we took the system (6.1) with various $D$ and initial condition $\left(\xi_{1}, \xi_{2}, \theta\right)=(1,1,0)$ and measured the proportion of time $\xi_{2}>1$ from $t=10^{3}$ to $t=10^{5}$.

In summary, the $\beta=0$ case is a system which switches intermittently between two quite distinct phases. It stays in the quiescent mode for an $O(1)$ time, then bursts for an $O(\ln (D) / D)$ time, with a burst height 


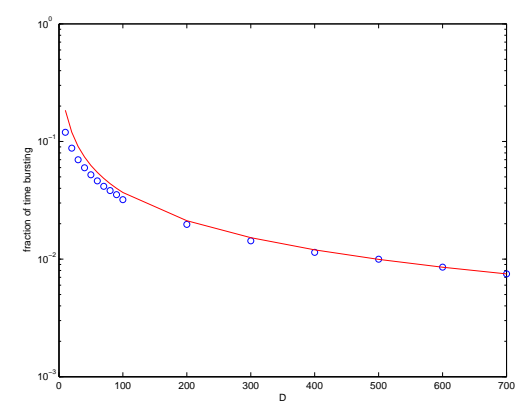

Figure 6.4. The solid curve is $0.7 \ln (D) / D$, and the circles are the proportion of time the system of (6.1) spends bursting as a function of $D$.

of $O(D)$. From this, one can calculate how the moments of $\xi_{2}$ act with respect to $D$. During the quiescent phase, $\xi_{2}$ moves between 0 and 1 like $e^{ \pm D t}$. The pdf of $\xi_{2}$ during this phase is $f\left(\xi_{2}\right)=\left(D \xi_{2}\right)^{-1}$. During the bursting phase, the solution moves from 1 to $O(D)$ in time $O(\ln (D) / D)$, so that the pdf in that phase is the uniform distribution $\ln (D) / D^{2}$. Therefore if we were to calculate the moments of $\xi_{2}$, we would have

$$
\begin{aligned}
\left\langle\xi_{2}^{n}\right\rangle & =\int_{0}^{1} d \xi_{2} \frac{\xi_{2}^{n-1}}{D}+\int_{1}^{D} d \xi_{2} \frac{\xi_{2}^{n} \ln (D)}{D^{2}} \\
& =D^{-1}+D^{n-1} \ln D .
\end{aligned}
$$

This shows that the two phases have quite different characters and explains why there is no uniform rescaling which can describe the statistics of both phases.

\section{Summary}

In this work, we have proposed a simple, two-mode model for nonlinear energy transfer, where one mode receives a controlled amount of energy through white noise, and the other dissipates energy through a nonlinear mechanism, which can be tuned to parameterize wave breaking.

The system exhibits a bifurcation between a quasi-equilibrium regime and one far from equilibrium as an appropriate non-dimensional combination of the driving and damping coefficients is increased. When the damping is linear, a case previously studied in [9], the thermal regime is Gaussian, and the non-equilibrium regime is highly intermittent. By contrast, under nonlinear damping, the equilibrium regime has non-Gaussian 
statistics, and the regime far from equilibrium is strongly constrained by slaving among the system's degrees of freedom.

\section{Acknowledgments}

This work was partially supported by NSF via grants DMS01-01439, DMS02-09959, DMS02-39625, DMS-0417732, DMS-0306444, and by ONR. Some of the results of this paper appeared in the R.J.P.'s PhD thesis [10].

\section{A Details of change of variables}

We do the $d \xi_{2}$ integral in (5.11) by making the substitution $\xi_{2}=\hat{E}-\xi_{1}$. Noting that the $d \xi_{2}$ integral is over positive reals, this means that we must have $\hat{E}-\xi_{1} \geq 0$, or $0 \leq \xi_{1} \leq \hat{E}$, and we get

$\int_{0}^{\infty} d \hat{E} \int_{-\hat{E}^{2} / 2}^{\hat{E}^{2} / 2} d \hat{H} \int_{0}^{2 \pi} d \theta \int_{0}^{\hat{E}} d \xi_{1} \delta\left(\gamma\left(\xi_{1}\right)-\hat{H}\right) F(H, \hat{E}) \phi\left(H, \hat{E}, \xi_{1}, \hat{E}-\xi_{1}, \theta\right)$,

where $\gamma\left(\xi_{1}\right)=H=2 \cos (2 \theta)\left(\hat{E} \xi_{1}-\xi_{1}^{2}\right)$. Let us for now consider the integral only in the $\hat{H}>0$ regime. Thus we consider

$\int_{0}^{\infty} d \hat{E} \int_{0}^{\hat{E}^{2} / 2} d \hat{H} \int_{-\pi / 4}^{\pi / 4} d \theta \int_{0}^{\hat{E}} d \xi_{1} \delta\left(\gamma\left(\xi_{1}\right)-\hat{H}\right) F(H, \hat{E}) \phi\left(H, \hat{E}, \xi_{1}, \hat{E}-\xi_{1}, \theta\right)$.

We only need to consider those $\theta$ for which $\cos (2 \theta)>0$, as the sign of $\hat{H}$ and the sign of $\cos (2 \theta)$ are the same. Let us note the following two symmetries of the integrand: If we make the change $\theta \mapsto-\theta$, every term in the integrand remains unchanged (every appearance of $\theta$ is as $\cos (2 \theta)$ ), and if we make the change $\xi_{1} \mapsto \hat{E}-\xi_{1}$, the terms $\gamma$ and $F$ remain unchanged. We exploit these symmetries, and can write the innermost two integrals as

$$
2 \int_{0}^{\pi / 4} d \theta \int_{0}^{\hat{E} / 2} d \xi_{1} \delta\left(\gamma\left(\xi_{1}\right)-\hat{H}\right) F(H, \hat{E})\left(\phi+\phi_{12}\right),
$$

where $\phi_{12}$ is $\phi$ with the roles of $\xi_{1}$ and $\hat{E}-\xi_{1}$ interchanged. Now we do the innermost integral, noting that $\gamma$ is invertible on this domain. Using the rule that

$$
\int_{a}^{b} d x \delta(\gamma(x)-A) f(x)=\frac{f\left(\gamma^{-1}(A)\right)}{\gamma^{\prime}\left(\gamma^{-1}(A)\right)} \text { if } A \in[\gamma(a), \gamma(b)]
$$


we get the innermost integral to be

$$
J_{1}(\hat{E}, \hat{H}, \cos (2 \theta)) F(\hat{H}, \hat{E})\left(\phi+\phi_{12}\right) \text { if } \cos (2 \theta) \geq \frac{2 \hat{H}}{\hat{E}^{2}},
$$

where

$$
J_{1}(\hat{E}, \hat{H}, \cos (2 \theta))=\frac{1}{2 \cos (2 \theta) \sqrt{\hat{E}-\frac{2 \hat{H}}{\cos (2 \theta)}}},
$$

and we use the substitution $\xi_{1} \mapsto \gamma^{-1}(\hat{H})=\frac{1}{2}\left(\hat{E}-\sqrt{\hat{E}^{2}-\frac{2 \hat{H}}{\cos (2 \theta)}}\right)$. Changing variables in the $d \theta$ integral using $\rho=\cos (2 \theta)$ brings (A.1) to

$$
\int_{1}^{\frac{2 \hat{H}}{E^{2}}} d \rho J_{2}(\hat{E}, \hat{H}, \rho) F\left(\phi+\phi_{12}\right),
$$

where

$$
J_{2}(\hat{H}, \hat{E}, \rho)=\frac{1}{2 \rho \sqrt{1-\rho^{2}} \sqrt{\hat{E}^{2}-\frac{2 \hat{H}}{\rho}}},
$$

and we make the further substitution in the integrand that $\cos (2 \theta) \mapsto \rho$.

Substituting $G=G(H, E)=G\left(2 \xi_{1} \xi_{2} \cos (2 \theta), \xi_{1}+\xi_{2}\right)$, (5.8) evaluates to

$$
\begin{aligned}
\phi=G_{E}(1- & \left.2 \xi_{2}^{\frac{\beta+2}{2}}\right)+G_{H}\left(-2 \xi_{1} \xi_{2}^{\frac{\beta+2}{2}} \cos (2 \theta)\right) \\
& +G_{E E} \xi_{1}+G_{H E}\left(4 \xi_{1} \xi_{2} \cos (2 \theta)\right)+G_{H H}\left(4 \xi_{1} \xi_{2}^{2}\right) .
\end{aligned}
$$

If we denote

$$
R_{ \pm}=\frac{1}{2}\left(\hat{E} \pm \sqrt{\hat{E}^{2}-\frac{2 \hat{H}}{\rho}}\right)
$$

note that after the necessary substitutions we have

$$
\begin{aligned}
\phi=G_{E}(1-2 & \left.R_{-}^{\frac{\beta+2}{2}}\right)+G_{H}\left(-2 R_{+} R_{-}^{\frac{\beta+2}{2}} \rho\right) \\
& +G_{E E} R_{+}+G_{H E} 4 R_{+} R_{-} \rho+G_{H H} 4 R_{+} R_{-} R_{-}, \\
\phi_{12}=G_{E}(1-2 & \left.R_{+}^{\frac{\beta+2}{2}}\right)+G_{H}\left(-2 R_{+}^{\frac{\beta+2}{2}} R_{-} \rho\right) \\
& +G_{E E} R_{-}+G_{H E} 4 R_{-} R_{+} \rho+G_{H H} 4 R_{+} R_{+} R_{-} .
\end{aligned}
$$

So, our integrand is $J_{2} F\left(\phi+\phi_{12}\right)$, with

$$
\begin{aligned}
\phi+\phi_{12}= & G_{E}\left(2-2\left(R_{-}^{\beta / 2+1}+R_{+}^{\beta / 2+1}\right)\right)+G_{H}\left(-H\left(R_{-}^{\beta / 2}+R_{+}^{\beta / 2}\right)\right) \\
& E G_{E E}+4 H G_{E H}+\frac{2 E H}{\rho} G_{H H} .
\end{aligned}
$$


If we define

$$
\begin{aligned}
J(\hat{H}, \hat{E}) & =\int_{2 \hat{H} / \hat{E}^{2}}^{1} J_{2}(\hat{H}, \hat{E}, \rho) d \rho \\
\phi_{-1}(\hat{H}, \hat{E}) & =\int_{2 \hat{H} / \hat{E}^{2}}^{1} \rho^{-1} J_{2}(\hat{H}, \hat{E}, \rho) d \rho \\
S_{\alpha}(\hat{H}, \hat{E}) & =\int_{2 \hat{H} / \hat{E}^{2}}^{1} J_{2}(\hat{H}, \hat{E}, \rho)\left(R_{+}(\hat{H}, \hat{E}, \rho)^{\alpha}+R_{-}(\hat{H}, \hat{E}, \rho)^{\alpha}\right) d \rho \\
\psi(\hat{H}, \hat{E}) & =\frac{\phi_{-1}}{J(\hat{H}, \hat{E})}, \quad T_{\alpha}(\hat{H}, \hat{E})=\frac{S_{\alpha}(\hat{H}, \hat{E})}{J(\hat{H}, \hat{E})}
\end{aligned}
$$

then we see that (A.2) becomes, after $d \rho$ integration,

$$
\begin{aligned}
J F\left\{\left(2-2 T_{\beta / 2+1}\right)\right. & G_{E}-\hat{H} T_{\beta / 2} G_{H} \\
& \left.+\hat{E} G_{E E}+4 \hat{H} G_{E H}+2 \hat{E} \hat{H} \psi G_{H H}\right\},
\end{aligned}
$$

which proves $(5.12)$. Also, note that $T_{0}(\hat{H}, \hat{E})=2$ and $T_{1}(\hat{H}, \hat{E})=\hat{E}$, so for $\beta=0$ this simplifies to

$$
J F\left\{2(1-E) G_{E}-2 H G_{H}+E G_{E E}+4 H G_{E H}+2 E H \psi G_{H H}\right\} .
$$

In general, however, $T_{\alpha}(\hat{H}, \hat{E})$ is a function of both $\hat{H}$ and $\hat{E}$, which means that for $\beta>0$, the drift coefficients depend on both variables.

\section{Bibliography}

[1] D. J. Benney and A. C. Newell. Random wave closures. Stud. in Appl. Math., 1969.

[2] D. J. Benney and P. G. Saffman. Nonlinear interactions of random waves in a dispersive medium. Proc. Roy. Soc. A, 1966.

[3] D. Cai, A. Majda, D. McLaughlin, and E. Tabak. Spectral bifurcations in dispersive wave turbulence. Proc. Nat. Acad. Sci., 1999.

[4] D. Cai, A. J. Majda, D. W. McLaughlin, and E. G. Tabak. Dispersive wave turbulence in one dimension. Phys. D, 152/153:551-572, 2001. Advances in nonlinear mathematics and science.

[5] A. D. D. Craik. Wave interactions and fluid flows. Cambridge Monographs on Mechanics and Applied Mathematics. Cambridge University Press, Cambridge, 1988.

[6] S. Dyachenko, A. C. Newell, A. Pushkarev, and V. E. Zakharov. Optical turbulence: weak turbulence, condensates and collapsing filaments in the nonlinear Schrödinger equation. Phys. D, 57(1-2):96-160, 1992. 
[7] K. Hasselmann. On the non-linear energy transfer in a gravity-wave spectrum. I. General theory. J. Fluid Mech., 12:481-500, 1962.

[8] A. J. Majda, D. W. McLaughlin, and E. G. Tabak. A one-dimensional model for dispersive wave turbulence. J. Nonlinear Sci., 7(1):9-44, 1997.

[9] P. A. Milewski, E. G. Tabak, and E. Vanden-Eijnden. Resonant wave interaction with random forcing and dissipation. Stud. Appl. Math., 108(1):123-144, 2002. Dedicated to Professor David J. Benney.

[10] R. Pignol. Energy Transfer in Systems with Random forcing and nonlinear dissipation. PhD thesis, Courant Institute of Mathematical Sciences, NYU, 2003.

[11] V. E. Zakharov, V. L'vov, and G. Falkovich. Wave Turbulence. Springer, 1992.

R. E. Lee DeVille

Courant Institute

251 Mercer Street

New York, NY 10012

E-mail: deville@cims.nyu.edu

Paul A. Milewski

Department of Mathematics

480 Lincoln Drive

University of Wisconsin

Madison, WI 10012

E-mail: milewski@math.wisc.edu

Ricardo J. Pignol

Departamento de Matemática

Universidad Nacional del Sur

Avda. Alem 1253 (8000)

Bahía Blanca, Argentina

Email: rpignol@criba.edu.ar

Esteban G. TABAK

Courant Institute

251 Mercer Street

New York, NY 10012

E-mail: tabak@cims.nyu.edu

ERIC VANDEn-EIJNDEN

Courant Institute

251 Mercer Street

New York, NY 10012

E-mail: eve2@cims.nyu.edu 